\title{
Aseguramiento de la calidad educativa universitaria en Iberoamérica: tendencias, ausencias y desafíos
}

\author{
Roberto Medina-Manrique ${ }^{1}$ (C) \\ Wilfredo Humberto Carcausto Calla ${ }^{1}$ \\ Evelin Elena Guzmán Shigetomi ${ }^{2}$ (D) \\ ${ }^{1}$ Universidad César Vallejo (UCV), Perú; ${ }^{2}$ Universidad Científica del Sur (UCSUR), Perú
}

Resumen. El aseguramiento de la calidad de la educación superior a nivel global es un proceso vital para lograr una cultura de mejora continua, en beneficio de la sociedad. Se busca proporcionar una visión sistemática de las tendencias y ausencias en el proceso de aseguramiento de calidad educativa universitaria de Iberoamérica, utilizando las dimensiones de calidad, según artículos científicos en revistas indizadas en dos bases de datos: Scopus y Scielo; entre 2012 y julio de 2021 . Se consideran como criterios: el año y país de publicación, las tendencias y ausencias en cada dimensión de calidad, y los desafíos propuestos. De los 85 artículos la mayor cantidad corresponde a España y Chile; la tendencia predominante es investigar sobre la dimensión de calidad del marco del aseguramiento interno y externo, y la principal ausencia es de artículos sobre la dimensión calidad en instalaciones, recursos académicos y soporte; con sólo $1 \%(n=1)$. Los desafíos en aseguramiento de la calidad son variados por cada dimensión, siendo algunos la educación online o virtual, que surge en 2013 pero vuelve a tener importancia por la pandemia de COVID 19, así como lograr una cultura de calidad e identidad, la homogenización versus la innovación, entre otros.

Palabras clave: aseguramiento de la calidad; educación superior universitaria; dimensiones de la calidad, desafíos.

Assegurar a qualidade do ensino universitário na Ibero-América: tendências, ausências e desafios

Resumo. A garantia da qualidade do ensino superior em nível global é um processo vital para alcançar uma cultura de melhoria contínua, em benefício da sociedade. Busca-se oferecer uma visão sistemática das tendências e ausências no processo de garantia da qualidade da educação universitária na IberoAmérica, utilizando as dimensões da qualidade, segundo artigos científicos em revistas indexadas em duas bases de dados: Scopus e Scielo; entre 2012 e julho de 2021. São considerados como critérios: o ano e o país de publicação, as tendências e ausências em cada dimensão da qualidade e os desafios propostos. Dos 85 artigos, o maior número corresponde à Espanha e ao Chile; a tendência predominante é pesquisar sobre a dimensão da qualidade da estrutura de garantia interna e externa, e a principal ausência é de artigos sobre a dimensão da qualidade nas instalações, recursos acadêmicos e suporte; com apenas $1 \%(n=1)$. Os desafios na garantia da qualidade são variados em cada dimensão, sendo alguns deles a educação on-line ou virtual, que surgiu em 2013, mas volta a ser importante devido à pandemia do COVID 19, além de alcançar uma cultura de qualidade e identidade, a homogeneização versus inovação, entre outros.

Palavras-chave: garantia da qualidade; ensino superior universitário; dimensões da qualidade, desafios

Assurance of university educational quality in Ibero-America: trends, absences and challenges

Abstract. The quality assurance of higher education globally is a vital process to achieve a culture of continuous improvement, for the benefit of society. It seeks to provide a systematic view of the trends and absences in the process of quality assurance of university education in Ibero-America, using quality dimensions, according to scientific articles in journals indexed in two databases: Scopus and Scielo; between 2012 and July 2021. The following are considered as criteria: the year and country of publication, the trends and gaps in each quality dimension, and the proposed challenges. Of the 85 articles, the largest quantity corresponds to Spain and Chile; The predominant trend is to investigate the quality dimension of the internal and external assurance framework, and the main absence is articles on the quality dimension in facilities, academic resources, and support; with only $1 \%(n=1)$. The challenges in quality assurance are varied for each dimension, some being online or virtual education, which emerged in 2013 but is again important due to the COVID 19 pandemic, as well as achieving a culture of quality and identity, homogenization versus innovation, among others.

Keywords: Quality assurance; university higher education; quality dimensions, challenges. 


\section{Introducción}

De acuerdo a UNESCO 40C (2019), UNESCO - IESALC, UNC (2018), la educación superior, dada de forma pública o privada, es considerada como un bien y una responsabilidad públicos, con libertad académica y autonomía, y es necesario asegurar su calidad. Esta calidad en educación superior se basa en tres elementos fundamentales: (1) la consistencia interna que determina la identidad de la institución siendo elemento clave el compromiso con los estudiantes, (2) la consistencia externa, de acuerdo con las demandas del entorno definiendo la pertinencia de sus actividades y (3) el ajuste de recursos y procesos internos de la institución y su identidad. En el escenario mundial, el aseguramiento de la calidad (AC) de la educación superior universitaria es de interés global, incluyendo Latinoamérica y España, por lo cual, a partir de los años 1990, se ha desarrollado estrategias de registro y de creación de estándares mínimos de evaluación y acreditación de las universidades. En este contexto, asegurar la calidad confronta a universidades y actores regulando la educación superior, cuestionando la capacidad de las primeras de dar formación a millones de estudiantes. De esta manera, el aseguramiento de la calidad universitaria a través de la autoevaluación y evaluación externa es la evidencia de su excelencia y sostenibilidad, generando la aparición de innovaciones pedagógicas, nuevos modelos de enseñanza-aprendizaje, respondiendo a la necesidad de elevar el nivel de formación del capital humano, (Rojas 2012; García-Jiménez 2016; Montesinos et al., 2019)

Por lo tanto, se define el término de aseguramiento de calidad, como el proceso continuo, diseñado y utilizado permanentemente mediante el cual se evalúa la calidad de un sistema, institución o programa de educación superior, asegurando a los interesados la mejora continua del nivel de calidad cumpliendo un conjunto de condiciones requeridas para realizar sus funciones, como una organización activa, cambiando y respondiendo al ambiente, proporcionando confianza a la sociedad (UNESCO 40C, 2019; y Adrogué et al., 2019). La heterogeneidad de la calidad en el mercado de la educación superior universitaria genera la creación de sistemas de acreditación, como una herramienta eficaz para mejorar los sistemas educativos en un contexto neoliberal, facilitando la legitimación y reconocimiento mutuo de las universidades como herramientas de impacto positivo en la sociedad (Díez, 2019; Jiménez, 2019). De esta manera se introducen mecanismos de aseguramiento internos y externos de la calidad que impactan directa e indirectamente en la universidad, creando una nueva cultura académica, cambiando radicalmente la comprensión de la enseñanza, gestión y todos los procesos de la educación superior, mejorando de manera continua los aprendizajes de los estudiantes como el fin último de dicha educación (Muñoz el al., 2013; Montecinos, 2019). Al aseguramiento de la calidad universitaria se asocia el termino de excelencia a las experiencias de enseñanza, aprendizaje e investigación de los actores, caracterizándose por indicadores de compromiso, rendimiento, satisfacción de los grupos de interés y responsabilidad social. (García-Jiménez, 2016). Además, promueve buenas prácticas gerenciales y una cultura de rendición de cuentas, por ser herramientas de desarrollo integral y bienestar económicos (Grifoll, 2016; Jiménez, 2019). 
De acuerdo con la Unesco 40C (2019) se debe promover una cultura de aseguramiento de la calidad de la educación superior y desarrollar las capacidades de manera fiable, consistente y complementaria, a partir de marcos de cualificación y reconocimiento de las cualificaciones, lo cual no ha sido investigado ni se tienen datos. Adicionalmente, en Iberoamérica, de acuerdo con las investigaciones encontradas, predomina la investigación del modelo de aseguramiento de la calidad netamente gerencial y de auditoría, con enfoque cuantitativo que toma como bandera la evaluación como instrumento de control, por encima del que conceptualiza la evaluación como instrumento de mejora, con un componente discrecional. (Marquina, 2017, Jacques-P. y Boisier-O, 2019). Se han encontrado, en español e inglés, pocas revisiones sistemáticas sobre el tema, solo cuatro en América Latina, la de Barreto y Kalnin (2018) que realiza una revisión teórica de la evolución del concepto de calidad en la educación superior por parte de autores brasileños e internacionales en el periodo 2005 -2015; la de Bernasconi y Celis, (2017) que analiza desde una perspectiva comparada las reformas iniciadas por los gobiernos latinoamericanos desde 1990 en políticas de calidad de educación superior; y la de Asencio et al. (2019) que realiza una revisión bibliográfica visión histórica, analítica, crítica de la calidad de educación médica en el Perú. A nivel global, encontramos la revisión sistemática de Ruiz-Ramírez y Glasserman-Morales (2021) que identifica las características descriptivas, a partir de 7 líneas temáticas, de los procesos de aseguramiento de la calidad educativa de 2016 a 2020 en dos bases de datos: Scopus y Web of Science (WoS) tomando artículos de 51 países, principalmente Estados Unidos, Malasia y Australia.

Por lo tanto, son necesarias investigaciones que sistematicen la importancia del aseguramiento de la calidad en la producción de artículos científicos reflejando la realidad en la educación universitaria iberoamericana como un problema multidimensional, (Varouchas et al., 2018), priorizando a los actores del proceso, con investigaciones que evidencian praxis culturales que busquen la trasformación de la persona y de su entorno. (González y Santamaría, 2013; García-Herrera, 2015).

Teniendo en cuenta que son desafíos del aseguramiento de la calidad las dimensiones que valoran aspectos cualitativos y que promuevan la conciencia acerca del aseguramiento de la calidad académica de las universidades (UNESCO 40C, 2019) es necesario conocer los componentes objetivos o de estructura (mirada puramente normativa, institucional, económica) y subjetivos como las percepciones individuales y colectivas de los actores en dicha estructura, incluyendo las relaciones interpersonales a través de la pertinencia, transparencia, percepción, participación, valorización social e involucramiento de estos actores, (Rojas, 2012; Jacques-P. y Boisier-O., 2019). Tomando en cuenta los tres elementos fundamentales de la calidad en educación superior, Varouchas et al. (2018) determinan cinco dimensiones de calidad en educación universitaria, que son: en consistencia interna: 1) enseñanza, aprendizaje y el currículo; 2) resultados de aprendizaje, conocimientos y habilidades de los graduados; en consistencia externa: 3) marco del aseguramiento; 4) sistema educativo del país; y en el ajuste de recursos y procesos: 5 ) instalaciones, recursos académicos y soporte

Por lo expuesto, el objetivo de esta investigación es proporcionar una visión sistemática de las tendencias y ausencias en el proceso de aseguramiento de calidad educativa universitaria de Iberoamérica, de acuerdo con las dimensiones de calidad, 
según los artículos científicos en revistas indizadas en dos bases de datos: Scopus y Scielo; entre 2012 y julio de 2021. Los objetivos específicos determinan la cantidad de artículos científicos originales 1) según año de publicación; 2) Según país de publicación; 3) Según las tendencias y ausencias en el tiempo de cada una de las cinco dimensiones de la calidad, 4) De acuerdo con los desafíos propuestos por dimensiones, y su evolución en el tiempo buscando la priorización de los actores del proceso, y la evidencia de praxis culturales que busquen la trasformación de los actores y del entorno. (González y Santamaría, 2013; García-Herrera, 2015).

\section{Metodología}

Para garantizar una revisión sistemática y exhaustiva de los estudios relevantes sobre el aseguramiento de la calidad educativa en educación superior en Iberoamérica se sigue el procedimiento definido por Patton (2018) que consta de cinco pasos: (1) identificar alcances y bases de datos, (2) establecer el estándar de selección, (3) definir los criterios de búsqueda (4) aplicar la búsqueda automatizada y (5) evaluar el rendimiento de la búsqueda y eliminación de duplicados determinando los artículos originales que se incluyen finalmente en la revisión.

\subsection{Procedimiento desarrollo de revisión sistemática de la literatura}

Identificar alcances y bases de datos. La revisión de la literatura se realizó para identificar y describir el estado de las tendencias, ausencias y desafíos en el tema de-aseguramiento de la calidad en educación superior universitaria, en artículos acceso abierto (open Access o OA) que se encuentran en las revistas indexadas de las bases de datos Scopus y Scielo.

En esta fase se seleccionó a los artículos científicos, los criterios de inclusión y-exclusión. En ese sentido, se incluyeron artículos de investigación originales sobre aseguramiento de la calidad en la educación universitaria en Iberoamérica de diez años de antigüedad, publicados entre 2012 y junio de 2021, con acceso abierto al texto completo, en idiomas español, inglés y portugués, con enfoque cualitativo o cuantitativo. Se excluyeron artículos que no tuvieran acceso abierto, publicados antes de 2012, estudios como libros, tesis, revisiones, cartas al editor, ensayos, resúmenes de conferencias, entre otros; referidos a países diferentes a Iberoamérica, en idiomas diferentes al español, inglés y portugués, y que no corresponden al nivel educativo universitario.

Definir los criterios de búsqueda. Se definió los criterios de búsqueda a través de la opción de título, usando el operador booleano AND para la selección preliminar, utilizando las combinaciones de palabras clave: "aseguramiento de calidad" AND "educación superior"; "quality assurance" AND "higher education".

Aplicar la búsqueda automatizada. En esta parte, se hizo la búsqueda automatizada de acuerdo con los criterios de inclusión y exclusión a través de los filtros de búsqueda avanzada, restringiendo la búsqueda a las palabras claves de la búsqueda o keywords, exportando la selección a Microsoft Excel ${ }^{\circledR}$.

Evaluar el rendimiento de la búsqueda. Para cumplir con el objetivo de la revisión sistemática, se identificó los artículos duplicados, para luego analizar los resúmenes y el texto completo del artículo, depurándolos para que los seleccionados cumplan 
con los objetivos y alcances. Luego se interpretaron los artículos para categorización de acuerdo con las dimensiones de la calidad educativa desarrolladas en el presente estudio para conocer los resultados sobre los alcances del estudio.

\section{Resultados}

En la tabla 1 se presenta los resultados de la aplicación de la metodología, identificando en la búsqueda por palabras clave en español (aseguramiento de calidad y educación superior) y en inglés (quality assurance and higher education) 21,473 en la base de datos Scopus, y 100 en la base de datos Scielo. Luego, se aplicó los criterios de inclusión y exclusión para la búsqueda automatizada en estas bases de datos ( $n=194$ en Scopus; y $n=25$ en Scielo).

Tabla 1. Operaciones booleanas de búsqueda para selección preliminar y final

\begin{tabular}{|c|c|c|c|c|}
\hline $\begin{array}{l}\text { Búsqueda por } \\
\text { palabras clave } \\
\text { en artículos }\end{array}$ & $\begin{array}{l}\text { Documen- } \\
\text { tos resul- } \\
\text { tantes }\end{array}$ & $\begin{array}{l}\text { Criterios de inclusión e exclusión para } \\
\text { búsqueda automatizada }\end{array}$ & $\begin{array}{c}\text { Resultado } \\
\text { preliminar } \\
\text { sin depu- } \\
\text { ración }\end{array}$ & $\begin{array}{l}\text { Resulta- } \\
\text { do final } \\
\text { depurado }\end{array}$ \\
\hline
\end{tabular}

Scopus
"quality assu-
rance" AND
"higher educa-
tion"

"aseguramiento de la calidad" AND "educación superior"
21,150 "quality assurance" AND "higher education" AND ( LIMIT-TO ( PUBSTAGE , "final")) AND ( LIMIT-TO ( OA, "all"

)) AND ( LIMIT-TO ( PUBYEAR , 2021 .. 2020 ... 2019 .. 2018 ... 2017 ... 2016 ... 2015 ... 2014 ... 2013 ... 2012 ) AND ( LIMIT-TO ( DOCTYPE, "ar" )) AND ( LIMIT-TO ( LANGUAGE , "English" .. "Spanish" .. "Portuguese" ) ) AND ( LIMIT-TO ( EXACTKEYWORD , "Higher Education") OR ... "Quality Assurance") ) AND ( LIMIT-TO (AFFILCOUNTRY, "Spain".. "Chile" .. "Portugal" .."Brazil" .. "Mexico" .. "Colombia" .. "Ecuador" .. "Guatemala" .. "Peru" .. "Argentina" .. , "Bolivia" .."Cuba" .. "Uruguay" ) )

323 "aseguramiento de la calidad" AND "educación superior" AND ( LIMIT-TO ( OA, "all")) AND ( LIMIT-TO ( PUBYEAR , 2021, LIMIT-TO ( PUBYEAR , $2020 \ldots ., 2019 \ldots 2018 \ldots 2017$... 2016 .. 2015 ... $2014 \ldots 2013$... 2012 ... 2011)) AND ( LIMIT-TO ( PUBSTAGE , "final")) AND ( LIMIT-TO ( DOCTYPE , "ar" )) AND ( LIMIT-TO ( LANGUAGE , "Spanish" ... "English" ..."Portuguese" )) AND ( LIMIT-TO ( AFFILCOUNTRY, "Chile" ... "Spain" ... "Mexico" ..."Colombia" .. "Ecuador" ..."Argentina" ... "Brazil" ..."Uruguay" ... "Bolivia" ..."Peru" ... "Portugal")) AND ( LIMIT-TO ( EXACTKEYWORD , "Quality Assurance") OR .. "Educacion Superior" )) 


\begin{tabular}{cccc}
\hline $\begin{array}{c}\text { Búsqueda por } \\
\text { palabras clave } \\
\text { en artículos }\end{array}$ & $\begin{array}{c}\text { Documen- } \\
\text { tos resul- } \\
\text { tantes }\end{array}$ & $\begin{array}{c}\text { Criterios de inclusión e exclusión para } \\
\text { búsqueda automatizada }\end{array}$ & $\begin{array}{c}\text { Resultado } \\
\text { preliminar } \\
\text { sesulta- } \\
\text { din depu- depurado } \\
\text { ración }\end{array}$ \\
\hline
\end{tabular}

\section{Scielo}

*aseguramiento de la calidad y educación superior

*quality assurance and higher education

\section{7}

*aseguramiento de la calidad y educación superior AND network:org AND -in:rve AND ( la:("es" OR "pt" OR "en") AND year_cluster: ("2020" OR "2012" OR "2019" OR "2016" OR "2018" OR "2011" OR “2017" OR "2021" OR “2015" OR "2013" OR "2014") AND subject_ area:(“Applied Social Sciences" OR "multidisciplinary"))

93 Filtros aplicados: (Idioma: Espanhol) (Idioma: Portugués) (Idioma: Inglés) (Ano de publicação: 2018, 2012, 2017, 2013, 2016, 2021, 2014, 2019) (Tipo de literatura: Artigo)

Fuente de organización de tabla: Ricoy y Sánchez-Martínez (2020). Elaboración: Propia.

6 18

Finalmente, en la figura 1 se aprecia el diagrama de flujo de la revisión sistemática realizada. Después de la depuración final se determina como resultado final 85 artículos ( $n=67$ en Scopus; y $n=18$ en Scielo) para proceder a la interpretación de acuerdo con las dimensiones de calidad educativa.

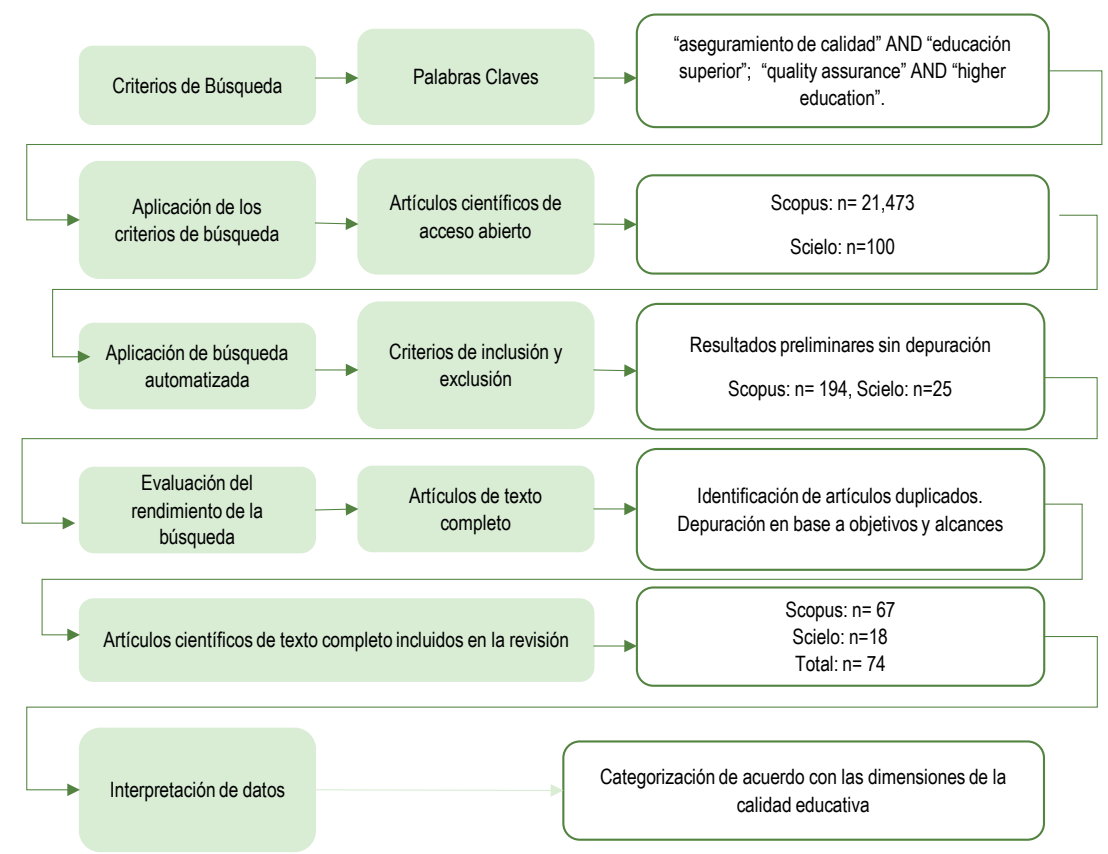

Figura 1. Diagrama de flujo para la revisión sistemática. Adaptado de Patton, Fluck, y Scanlan (2018) 
De acuerdo con el número de publicaciones por año sobre aseguramiento de la calidad en Iberoamérica, decrecen desde el 2012 al 2015 (en este año solo hay una publicación en Scopus). A partir de 2016 empieza una tendencia creciente, y al mes de junio de 2021, se percibe que continua esta tendencia lo que podría revelar un aumento de la importancia del tema para los investigadores iberoamericanos. El mayor número de artículos (14 artículos) se produce en 2020, siendo 13 de estos de la base de datos Scopus. En la base de datos Scielo, el 2018 fue el año con mayor producción.

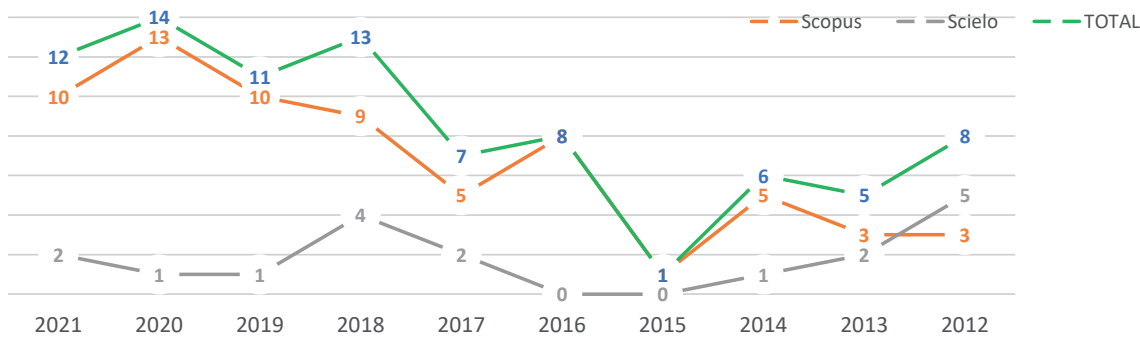

Figura 2. Artículos sobre aseguramiento de calidad en educación superior publicados en Iberoamérica por año y base de datos.

Si analizamos las cantidades con mayor producción de artículos científicos sobre aseguramiento de calidad en educación superior universitaria, el mayor porcentaje (29\%) corresponden a España con 33\% ( $n=28)$. Le sigue Chile con $21 \%(n=18)$ y Colombia con $10 \%(n=9)$. También encontramos investigaciones que corresponden a ámbitos más amplios (Iberoamérica, Latinoamérica y Sudamérica).

Tabla 2. Investigaciones en Iberoamérica del 2012 al 2021 por país y base de datos

\begin{tabular}{lrrrr}
\hline \multicolumn{1}{c}{ Base de datos } & SCOPUS & SCIELO & TOTAL & $\%$ \\
\cline { 2 - 3 } \multicolumn{1}{c}{ País } & 5 & & 5 & 6 \\
Argentina & 1 & 4 & 5 & 6 \\
Brasil & 12 & 6 & 18 & 21 \\
Chile & 8 & 1 & 9 & 10 \\
Colombia & 1 & & 1 & 1 \\
Costa Rica & & 3 & 3 & 3 \\
Cuba & 4 & 1 & 5 & 6 \\
Ecuador & 27 & 1 & 28 & 33 \\
España & 3 & 1 & 4 & 5 \\
México & 2 & & 2 & 2 \\
Perú & 2 & & 2 & 2 \\
Portugal & 1 & & 1 & 1 \\
Iberoamérica & 1 & 1 & 1 & 1 \\
Latinoamérica & & & 1 & 1 \\
Sudamérica & 68 & 21 & 100 & 100 \\
TOTAL & 79 & & & \\
\% & & & & \\
\hline
\end{tabular}

Fuente: elaboración propia 
Para análisis interpretativo de las tendencias y ausencias por dimensión de calidad, cada artículo fue revisado por los autores y clasificados de acuerdo con su objetivo principal y conclusiones. De acuerdo con esta metadata generada, (acá iría el link al cuadro de metadata) fue posible identificar y ordenar los artículos por dimensiones de la calidad educativa, como se muestra en la tabla 3.

En el periodo 2012-2021, se presenta una tendencia predominante de la dimensión 1 (calidad en el marco del aseguramiento interno y externo), con 46\% ( $n=39)$, seguido de los artículos de la dimensión 2 (calidad en la enseñanza, el aprendizaje y el currículo), con 25\% ( $n=21)$, y de la dimensión 3 (calidad del sistema educativo del país) con 19\% $(n=16)$. Además, se revela como ausencias significativas las investigaciones sobre la dimensión 4 (calidad de los resultados de aprendizaje, conocimientos y habilidades), con $9 \%(n=8)$ y, sobre todo, la mínima preocupación por investigar en la dimensión 5 (calidad en instalaciones, recursos académicos y soporte); con sólo $1 \%(n=1)$

Tabla 3. Clasificación de los artículos por dimensiones de la calidad

\begin{tabular}{|c|c|c|c|}
\hline Dimensión & $\begin{array}{l}\text { Intervalo de } \\
\text { Artículos }\end{array}$ & Total $(n)$ & $\begin{array}{l}\text { Porcentaje } \\
(\%)\end{array}$ \\
\hline $\begin{array}{l}\text { Calidad en el marco de aseguramiento interno y exter- } \\
\text { no }\end{array}$ & 1 al 39 & 39 & 46 \\
\hline Calidad en la enseñanza, el aprendizaje y el currículo; & 40 al 60 & 21 & 25 \\
\hline Calidad del sistema educativo del país; & 61 al 76 & 16 & 19 \\
\hline $\begin{array}{l}\text { Calidad en resultados de aprendizaje, conocimientos y } \\
\text { habilidades de los graduados. }\end{array}$ & 77 al 84 & 8 & 9 \\
\hline $\begin{array}{l}\text { Calidad en instalaciones, recursos académicos y so- } \\
\text { porte; }\end{array}$ & 85 & 1 & 1 \\
\hline Total & & 85 & 100 \\
\hline
\end{tabular}

Fuente: elaboración propia

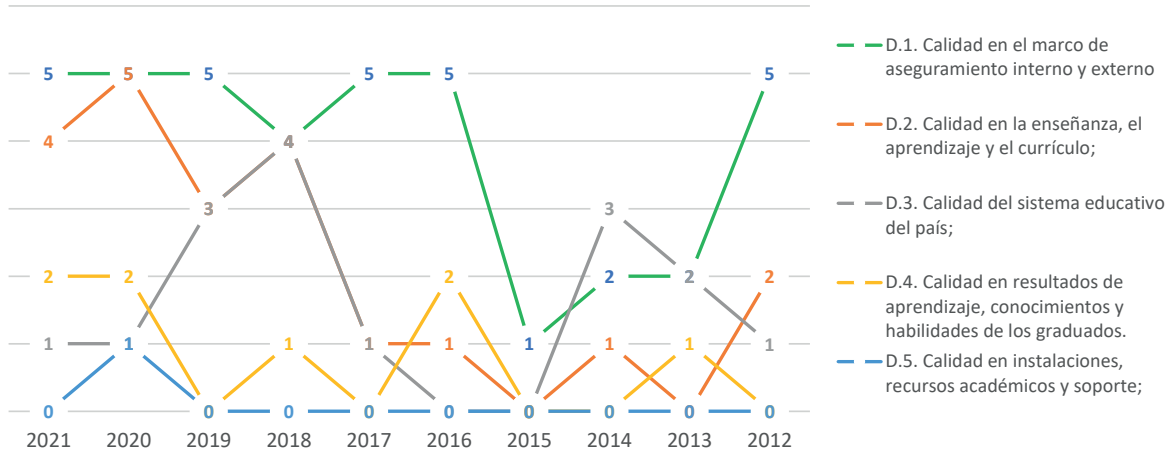

Figura 3. Cantidad de Investigaciones por dimensiones de la calidad

Las tres dimensiones indicadas como tendencias, a diferencia de las ausencias encontradas, no se desarrollan de manera homogénea en los años del intervalo estudiado. Las dimensiones 1 y 2 presentan actualmente una tendencia creciente, 
sin embargo, pueden ser catalogadas como ausencias entre los años 2013 a 2015; a diferencia de la dimensión 3, con una tendencia creciente del 2012 al 2014, que se repite entre el 2017 y 2018, pero luego decrece en 2019 y se convierte en una ausencia entre el 2020 y 2021. La dimensión 4, catalogada como ausencia por la baja cantidad de investigaciones en los últimos 10 años, presenta tendencias crecientes en 2016 y en los últimos 2 años.

Las dimensiones establecidas permiten analizar los principales desafíos que plantean las investigaciones desarrolladas en los artículos por años, determinando categorías estratégicas sobre el aseguramiento de la calidad de acuerdo con los resultados y conclusiones generados. Se puede apreciar la variación de dichas categorías en el tiempo. Las categorías de mayor relevancia encontradas son: En la dimensión 1 educación virtual (con sus variantes no presencial y a distancia), toma de decisiones, identidad y cultura institucional, excelencia, estándares de calidad; en la dimensión 2 la satisfacción de estudiantes; en la dimensión 3 las regulaciones, la validez y legitimidad, las políticas educativas; en la dimensión 4 la satisfacción de los graduados; y en la dimensión 5 la calidad del gasto y las mejoras en infraestructura y equipos.

Tabla 4. Desafíos sobre aseguramiento de la calidad por dimensiones en investigaciones de Iberoamérica del 2012 al 2021

\begin{tabular}{|c|c|c|c|c|c|}
\hline Años & $\begin{array}{c}\text { Marco de } \\
\text { aseguramiento } \\
\text { interno y externo }\end{array}$ & $\begin{array}{l}\text { Enseñanza, el } \\
\text { aprendizaje y el } \\
\text { currículo }\end{array}$ & $\begin{array}{l}\text { Sistema } \\
\text { educativo } \\
\text { del país }\end{array}$ & $\begin{array}{c}\text { Resultados de } \\
\text { aprendizaje, } \\
\text { conocimientos } \\
\text { y habilidades } \\
\text { de los } \\
\text { graduados }\end{array}$ & $\begin{array}{l}\text { Instalaciones, } \\
\text { recursos } \\
\text { académicos } \\
\text { y soporte }\end{array}$ \\
\hline 2021 & $\begin{array}{l}\text { Integración de } \\
\text { estándares de } \\
\text { calidad; Protección } \\
\text { de grupos vulne- } \\
\text { rables; Acompaña- } \\
\text { miento socioemo- } \\
\text { cional por covid19 } \\
\text { ID: } 1 ; 2 ; 3 ; 4 ; 5\end{array}$ & $\begin{array}{l}\text { Actores y atribu- } \\
\text { tos en enseñanza } \\
\text { online. } \\
\text { Satisfacción; Moti- } \\
\text { vación; Reformas. } \\
\text { ID: } 40 ; 41 ; 42 ; 43\end{array}$ & $\begin{array}{l}\text { Validez - } \\
\text { Apropiación } \\
\text { por los } \\
\text { actores. } \\
\text { ID: } 61\end{array}$ & $\begin{array}{l}\text { Sostenibilidad, } \\
\text { innovación y } \\
\text { género. } \\
\text { Mejora de } \\
\text { desempeño. } \\
\text { ID: } 77 ; 78\end{array}$ & $\begin{array}{l}\text { * Infraes- } \\
\text { tructura en } \\
\text { el marco del } \\
\text { asegura- } \\
\text { miento } \\
\text { ID:3 }\end{array}$ \\
\hline 2020 & $\begin{array}{l}\text { Factores de éxito } \\
\text { Sostenibilidad } \\
\text { Educación virtual. } \\
\text { Información para } \\
\text { toma de decisio- } \\
\text { nes (2) } \\
\text { ID:6; 7; 8; 9; } 10\end{array}$ & $\begin{array}{l}\text { Satisfacción de es- } \\
\text { tudiantes y reputa- } \\
\text { ción institucional. } \\
\text { Simplificación del } \\
\text { proceso. } \\
\text { Capacitación do- } \\
\text { cente. } \\
\text { Familia. } \\
\text { Sostenibilidad. } \\
\text { ID: } 44 ; 45 ; 46 ; \\
47 ; 48\end{array}$ & $\begin{array}{l}\text { Criterios } \\
\text { comunes de } \\
\text { cobertura } \\
\text { ID: } 62\end{array}$ & $\begin{array}{l}\text { Compromiso } \\
\text { institucional. } \\
\text { Satisfacción } \\
\text { de graduados. } \\
\text { ID: } 79 ; 80\end{array}$ & $\begin{array}{l}\text { Calidad del } \\
\text { gasto y me- } \\
\text { jora de las } \\
\text { condiciones } \\
\text { ID:85 }\end{array}$ \\
\hline
\end{tabular}




\begin{tabular}{|c|c|c|c|c|c|}
\hline Años & $\begin{array}{c}\text { Marco de } \\
\text { aseguramiento } \\
\text { interno y externo }\end{array}$ & $\begin{array}{l}\text { Enseñanza, el } \\
\text { aprendizaje y el } \\
\text { currículo }\end{array}$ & $\begin{array}{l}\text { Sistema } \\
\text { educativo } \\
\text { del país }\end{array}$ & $\begin{array}{l}\text { Resultados de } \\
\text { aprendizaje, } \\
\text { conocimientos } \\
\text { y habilidades } \\
\text { de los } \\
\text { graduados }\end{array}$ & $\begin{array}{l}\text { Instalaciones, } \\
\text { recursos } \\
\text { académicos } \\
\text { y soporte }\end{array}$ \\
\hline 2019 & $\begin{array}{l}\text { Identidad y cultura } \\
\text { institucional (2) } \\
\text { Masificación } \\
\text { Pertinencia y apro- } \\
\text { piación } \\
\text { ID: } 11 ; 12 ; 13 \text {; } \\
\text { 14; } 15\end{array}$ & $\begin{array}{l}\text { Compromiso do- } \\
\text { cente. } \\
\text { Despersonaliza- } \\
\text { ción. } \\
\text { Participación de } \\
\text { estudiantes y auto- } \\
\text { satisfacción. } \\
\text { Mejora adminis- } \\
\text { trativa. } \\
\text { ID: } 49 ; 50 ; 51\end{array}$ & $\begin{array}{l}\text { Política de } \\
\text { educación } \\
\text { virtual. Con- } \\
\text { venios. } \\
\text { Depen- } \\
\text { dencia de } \\
\text { recursos } \\
\text { económi- } \\
\text { cos. } \\
\text { ID: } 63 ; 64 ; \\
65\end{array}$ & & $\begin{array}{l}\text { *Recursos } \\
\text { financieros } \\
\text { para infraes- } \\
\text { tructura y } \\
\text { equipos } \\
\text { ID;15 }\end{array}$ \\
\hline 2018 & $\begin{array}{l}\text { Competitividad } \\
\text { Aprendizaje insti- } \\
\text { tucional } \\
\text { Profundidad de los } \\
\text { cambios } \\
\text { Utilidad } \\
\text { ID: 16; 17; 18; } 19\end{array}$ & $\begin{array}{l}\text { Innovación multidi- } \\
\text { mensional. } \\
\text { Tutoría. } \\
\text { Satisfacción de } \\
\text { estudiantes. } \\
\text { Aprendizaje signifi- } \\
\text { cativo. } \\
\text { ID: } 52 ; 53 ; 54 ; 55\end{array}$ & $\begin{array}{l}\text { Misiones } \\
\text { por cada } \\
\text { universidad. } \\
\text { Planifica- } \\
\text { ción de } \\
\text { políticas. } \\
\text { Crítica a } \\
\text { políticas } \\
\text { centraliza- } \\
\text { das. } \\
\text { ID: } 66 ; 67 ; \\
68 ; 69\end{array}$ & $\begin{array}{l}\text { Homogeniza- } \\
\text { ción vs inno- } \\
\text { vación } \\
\text { ID: } 81\end{array}$ & \\
\hline 2017 & $\begin{array}{l}\text { Liderazgo } \\
\text { Características } \\
\text { socioeconómicas } \\
\text { de estudiantes } \\
\text { Cultura de autorre- } \\
\text { gulación y autoe- } \\
\text { valuación } \\
\text { Lucro y calidad } \\
\text { ID: } 20 ; 21 ; 22 ; \\
23 ; 24\end{array}$ & $\begin{array}{l}\text { Currículo por com- } \\
\text { petencias } \\
\text { ID: } 56\end{array}$ & $\begin{array}{l}\text { Regulacio- } \\
\text { nes } \\
\text { ID: } 70\end{array}$ & & \\
\hline 2016 & $\begin{array}{l}\text { Excelencia (2). } \\
\text { Retos de la univer- } \\
\text { salización } \\
\text { Educación virtual } \\
\text { (2) } \\
25 ; 26 ; 27 ; 28 ; 29\end{array}$ & $\begin{array}{l}\text { Enfoque de genero } \\
\text { ID: } 57\end{array}$ & & $\begin{array}{l}\text { Competencias } \\
\text { genéricas } \\
\text { Inversión y } \\
\text { calidad de } \\
\text { graduados. } \\
\text { ID: } 82 ; 83\end{array}$ & \\
\hline 2015 & $\begin{array}{l}\text { Rentabilidad y } \\
\text { niveles heterogé- } \\
\text { neos de calidad. } \\
\text { ID: } 30\end{array}$ & & & & \\
\hline
\end{tabular}




\begin{tabular}{|c|c|c|c|c|c|}
\hline Años & $\begin{array}{c}\text { Marco de } \\
\text { aseguramiento } \\
\text { interno y externo }\end{array}$ & $\begin{array}{l}\text { Enseñanza, el } \\
\text { aprendizaje y el } \\
\text { currículo }\end{array}$ & $\begin{array}{c}\text { Sistema } \\
\text { educativo } \\
\text { del país }\end{array}$ & $\begin{array}{l}\text { Resultados de } \\
\text { aprendizaje, } \\
\text { conocimientos } \\
\text { y habilidades } \\
\text { de los } \\
\text { graduados }\end{array}$ & $\begin{array}{l}\text { Instalaciones, } \\
\text { recursos } \\
\text { académicos } \\
\text { y soporte }\end{array}$ \\
\hline 2014 & $\begin{array}{l}\text { Contexto social. } \\
\text { Educación no } \\
\text { presencial } \\
33 ; 34\end{array}$ & $\begin{array}{l}\text { Impacto en activi- } \\
\text { dades académicas } \\
\text { ID: } 58\end{array}$ & $\begin{array}{l}\text { Regulacio- } \\
\text { nes y auto- } \\
\text { nomía. } \\
\text { Certificacio- } \\
\text { nes. } \\
\text { Legitimidad. } \\
\text { ID: } 71 ; 72 ; \\
73\end{array}$ & & \\
\hline 2013 & $\begin{array}{l}\text { Educación a dis- } \\
\text { tancia. } \\
\text { Cumplimiento de } \\
\text { indicadores } \\
\text { ID: } 33 ; 34\end{array}$ & & $\begin{array}{l}\text { Conflicto } \\
\text { con merca- } \\
\text { do. } \\
\text { Visibilidad } \\
\text { a través de } \\
\text { la investiga- } \\
\text { ción. } \\
\text { ID: } 74 ; 75\end{array}$ & $\begin{array}{l}\text { Satisfacción } \\
\text { de graduados } \\
\text { ID:84 } \\
\text { ID: } 84\end{array}$ & \\
\hline 2012 & $\begin{array}{l}\text { Incidencia en } \\
\text { actores } \\
\text { Confusión entre } \\
\text { proceso y logro } \\
\text { Posicionamiento } \\
\text { Estándares de } \\
\text { calidad (2) } \\
\text { ID: } 35 ; 36 ; 37 ; \\
38 ; 39\end{array}$ & $\begin{array}{l}\text { Homogenización } \\
\text { como riesgo. } \\
\text { Profesionalidad y } \\
\text { calidad percibida. } \\
\text { ID: } 59 ; 60\end{array}$ & $\begin{array}{l}\text { Equidad } \\
\text { ID: } 76\end{array}$ & & \\
\hline
\end{tabular}

Leyenda: ID es la abreviatura que identifica el número designado a los autores.

*Se han encontrado en artículos cuya dimensión principal es otra

\section{Discusión}

En Iberoamérica, se evidencia por el número de artículos publicados que ha aumentado la importancia de la investigación y publicación sobre el aseguramiento de la calidad, el cual sigue siendo un tema controversial por su multidimensionalidad, su conceptualización como proceso, producto o meta, así como por el involucramiento de indicadores cualitativos como satisfacción, excelencia, autoevaluación e internacionalización

Respecto a los países con mayor producción intelectual, España ocupa el primer lugar, con investigaciones sobre el análisis y cuestionamiento de la oportunidad de establecer reformas abiertas por el proceso de Bolonia y la integración al proceso de aseguramiento de calidad como parte de Espacio Común Europeo de Educación Superior. Chile ocupa el segundo lugar, presentando publicaciones sobre la evolución de la política de calidad y su complejidad, producto de la realidad universitaria que cambia en 1999 con el sistema de acreditación de la educación superior y luego con la Comisión Nacional de Acreditación, donde se percibe una gran diversidad de 
calidad de la oferta universitaria, tanto en pregrado como en posgrado; y se cuestionan los efectos de las medidas de aseguramiento de la calidad que han llevado a la despersonalización de la educación y el mantenimiento del paradigma centralista de las políticas públicas chilenas.

De acuerdo con las tendencias encontradas en cada una de las dimensiones de la calidad, el mayor número de artículos se refieren a la dimensión de aseguramiento interno y externo de la calidad, especialmente en la temática de la educación virtual y su calidad, así como la inclusión a los modelos de aseguramiento de calidad de programas de conceptos como identidad, sentido de pertenencia y apropiación del proceso, por parte de los actores de estos modelos. Además, un concepto que se introduce en el 2020 es la sostenibilidad como un factor determinante de los modelos de evaluación de calidad de las universidades, que se relaciona con la satisfacción como ventaja competitiva.

En la dimensión de calidad de la enseñanza, el aprendizaje y el currículo, se considera de gran relevancia el análisis de la percepción de satisfacción de los diversos grupos de interés sobre la calidad de la educación superior, identificando atributos que permitan a las universidades mejorar la interacción docente - alumno, e incorporando métodos participativos en el proceso de enseñanza aprendizaje, así como la calidad percibida.

Respecto a la dimensión de la calidad del sistema educativo del país, se prioriza las evaluaciones y balances de la aplicación de políticas de aseguramiento de calidad, y su apropiación por los actores como instrumentos legítimos de mejora a ser evaluados a largo plazo. Esto asegura los procesos de calidad que son regulados por los organismos de fiscalización, sin olvidar los conflictos con el mercado y la búsqueda de igualdad de oportunidades y equidad. Es importante la presencia de los temas de productividad científica, y de la debilidad de las políticas por la dependencia de los recursos económicos.

Respecto a la dimensión de calidad en los resultados de aprendizaje, conocimientos y habilidades de los graduados, el principal tema investigado es la competitividad, satisfacción y compromiso de estos, demostrando sostenibilidad e innovación de la mejora curricular y analizando la adecuación del aseguramiento de la calidad universitaria a las necesidades del mercado laboral.

Por otro lado, resulta relevante la ausencia de artículos publicados en la dimensión de instalaciones, recursos académicos y soporte, a pesar de ser necesarios para el aseguramiento de la calidad. Solo en Cuba, investigan sobre la calidad del gasto y las condiciones académicas. Sin embargo, es componente de investigaciones que mencionan la infraestructura educativa en la primera dimensión.

De acuerdo con el análisis interpretativo de los desafíos planteados por las conclusiones y recomendaciones de los artículos sobre aseguramiento de la calidad en educación superior, se resaltan las categorías relacionadas con la educación online o virtual, que surge en el 2013 y 2014, y que vuelve a plantearse en los últimos años (2019 a 2021) por el impacto de la pandemia de COVID 19. Como se puede apreciar, los desafíos varían desde la aplicación general de los estándares de aseguramiento de calidad, la confusión entre el proceso y el logro de dicha calidad, las regulaciones 
políticas y su impacto en las actividades académicas (entre el 2012 al 2015), evolucionando del 2017 a 2019 a la generación de una cultura de calidad e identidad, a partir de la autorregulación y el aprendizaje institucional, criticando las políticas centralizadas, planteando el debate entre la homogenización versus la innovación y la necesidad de recursos financieros. En el momento actual (2020 y 2021) las categorías que emergen son el compromiso institucional de la sostenibilidad del aseguramiento de la calidad en la educación superior buscando la satisfacción y motivación de los actores, protegiendo grupos vulnerables a través de apoyos en la coyuntura de la educación virtual por el COVID 19.

\section{Conclusiones}

En síntesis, la revisión sistemática muestra que el número de artículos publicados sobre aseguramiento de la calidad en educación superior universitaria en Scopus y Scielo sobre Iberoamérica es baja, pero tiene una tendencia creciente, sobre todo a partir del año 2018, siendo España y Chile, los países sobre cuyas experiencias se trata en la producción científica tomada en este artículo, de acuerdo con los parámetros de búsqueda ejecutados. Los demás países no tienen un número representativo de investigaciones para conocer su realidad en el tema.

Al ser la calidad y su aseguramiento un concepto multidimensional, encontramos que, de los 85 artículos seleccionados, el 46\% tratan principalmente de la dimensión de calidad en el marco del aseguramiento interno y externo, principalmente la temática de los modelos de aseguramiento y los métodos de evaluación y autoevaluación. Se tiene poco número de artículos sobre la dimensión de la calidad en resultados de aprendizaje, conocimientos y habilidades de los graduados (9\%), y un 1\% de desarrollo de investigaciones sobre la dimensión de calidad en instalaciones, recursos académicos y soporte.

Por último, es importante observar la evolución de los desafíos en aseguramiento de calidad, donde se aprecian temas recurrentes que se repiten durante los 10 años transcurridos, lo que evidencia que el tema del aseguramiento de la calidad no ha sido cerrado, ni depende de procesos determinados por la política nacional, ni por estándares o indicadores dados, sino que depende de la cultura organización donde resalta la percepción, motivación y satisfacción de los actores de cada institución, como respuesta a intentos de homogenización; además surge el tema de género y de la educación virtual por factores que complejizan los criterios a aplicar en Iberoamérica.

\section{Limitaciones y prospectiva}

La principal limitación es la selección de artículos publicados sobreaseguramiento de la calidad en educación superior universitaria en dos bases de datos, aunque muy relevantes en Iberoamérica, como Scopus y Scielo. Por ello, un posible abordaje futuro es incluir en el análisis revistas indexadas con mayor reconocimiento como Web of Science. Además, sería importante realizar correlaciones entre las tendencias de las dimensiones de calidad con los artículos de cada país. 


\section{Referencias}

Adrogué, C., de Fanelli, A. G., Carranza, M. P. y Salto, D. J. (2019). Las universidades frente al aseguramiento de la calidad y las políticas de financiamiento de la investigación: estudios de caso en el sector privado argentino. Revista de La Educacion Superior, 48(190), 45-70. Accesible en https://bit.ly/34f7El5

Asencio, A., Barrios, Y., Chuquihuara, A, de la Cruz, D. y Munares, O. (2019). Análisis crítico e histórico de la calidad de educación médica en el Perú. Educación Médica Superior, 33(1), e1413. Recuperado de https://bit.ly/3KOcpmn.

Barreto, M. C. y Kalnin, G. F. (2018). Quality assurance: A theoretical review of its conceptual evolution in higher education. [Qualidade na educação superior: Uma revisão teórica da evolução conceitual no campo da educação superior] Ensaio, 26(100), 530-551. https://doi. org/10.1590/S0104-40362018002601114

Bernasconi, A. y Celis, S. (2017). Higher education reforms: Latin America in comparative perspective. [Reforma do ensino superior: América Latina em perspectiva comparada; Reformas de la educación superior: América Latina en perspectiva comparada] Education Policy Analysis Archives, 25. https://doi.org/10.14507/epaa.25.3240.

Díez, A. B. (2019). Structural problems of the accreditation of higher education in Chile: 2006-2012. [Problemas estructurales de la acreditación de la educación superior en Chile: 20062012] Revista Pedagogía Universitaria y Didáctica del Derecho, 6(1), 43-76. https://doi. org/10.5354/0719-5885.2019.53745

García Herrera, A. (2015). “Crisis y transformación de la educación superior: el lugar de las humanidades en Latinoamérica". Literatura: teoría, historia, crítica, 17(2), 79-96.

García-Jiménez, E. (2016). A definition of excellence in higher education. [Concepto de excelencia en enseñanza superior universitaria] Educación Médica, 17(3), 83-87.https://doi.org/10.1016/j. edumed.2016.06.003.

González J. y Santamaría, R. (2013). Calidad y acreditación en la educación superior: integración e internacionalización de América Latina y el Caribe. Educación 22(43), 131-147. Disponible en https://bit.ly/3ubFMJg

Grifoll, J. (2016). External quality assurance agencies and excellence in higher education. [Las agencias de aseguramiento externo de la calidad y la excelencia en educación superior] Educación Médica, 17(3), 94-96. https://doi.org/10.1016/j.edumed.2016.08.001

Jacques-P., V. y Boisier-O. G. (2019). La calidad en las instituciones de educación superior. Una mirada crítica desde el institucionalismo. Revista Educación, 43(1). Recuperado de https://bit.ly/3rR668I

Jiménez, J. A. J. (2019). The evaluation and accreditation of professional education in Mexico: Legitimacy and competitiveness as the goal of the university? [La evaluación y acreditación de la educación profesional en México: ¿la legitimación y competitividad como fin de la universidad?]. Revista de la Educación Superior, 48(189), 55-72. https://doi.org/10.36857/ resu.2019.189.614.

Marquina, M. (2017). Equilibrios y tensiones en dos décadas de acreditación universitaria en Argentina: aportes para un modelo conceptual. Revista Educación, 41(2). Recuperado de https:// bit.ly/32CloFQ.

Montecinos, M. V., Toledo, M. B., Amaya, W. S., Briceño, M. H. y Castillo, S. C. (2019). Quality assurance in virtual education. an analysis from public policies in Chile. [Aseguramiento de la calidad en la educación virtual. Un análisis desde las políticas públicas en chile]. Opción, 35(90), 1465-1490. Accesible en https://bit.ly/3g4OTTZ.

Muñoz, C. P., Nieto, B. B., Méndez, M. J. M., Morillejo, E. A y Rodríguez, M. P. G. (2013). Monitoring of official degree qualifications: The key role of academic quality assurance systems (AQAS). Universitas Psychologica, 12(1), 209-220. https://doi.org/10.11144/javeriana. upsy12-1.modq 
Patton, R. M., Fluck, A. E. \& Scanlan, J. D. (2018). Engagement and retention in VET MOOCs and online courses: A systematic review of literature from 2013 to 2017. Computers and Education, 125, 191-201. https://doi.org/10.1016/j.compedu.2018.06.013.

Ricoy, M. y Sánchez-Martínez, C. (2020). Revisión sistemática sobre el uso de la tableta en la etapa de educación primaria. Revista Española de Pedagogía, 78(276), 273-290. https://doi. org/10.22550/REP78-2-2020-04

Rojas, E. T. (2012). Institutional accreditation and the actors' perception. A qualitative study in private universities of Santiago. [Acreditación institucional y la mirada de los actores. Un estudio cualitativo en universidades privadas de Santiago] Estudios Pedagógicos, 38(2), 221-242. https://doi.org/10.4067/S0718-07052012000200014

Ruiz-Ramírez, J. y Glasserman-Morales, L. (2021). Características del aseguramiento de la calidad educativa: Un mapeo sistemático 2016-2020. Revista Complutense de Educación, 32(3), 337-348. https://doi.org/10.5209/rced.70182.

UNESCO - IESALC, UNC (2018). La educación superior como parte del sistema educativo de América Latina y el Caribe. Calidad y aseguramiento de la calidad. Recuperado de https://www. iesalc.unesco.org. ve

UNESCO 40C (2019). Informe sobre los progresos realizados en la preparación del proyecto de convención mundial sobre el reconocimiento de las cualificaciones relativas a la educación superior. Conferencia General 40ª reunión - Paris, 2019.

Varouchas, E., Sicilia, M., \& Sánchez-Alonso, S. (2018). Academics' perceptions on quality in higher education shaping key performance indicators. Sustainability (Switzerland), 10(12) https:// doi.org/10.3390/su10124752

\section{Cómo citar en APA:}

Medina-Manrique, R., Carcausto, W. H. y Guzmán, E. E. (2022). Aseguramiento de la calidad educativa universitaria en Iberoamérica: tendencias, ausencias y desafíos. Revista Iberoamericana de Educación, 88(1), 33-47. https://doi.org/10.35362/rie8814774 